\title{
Gesellschaftlicher Nutzen der Adipositaschirurgie
}

\author{
Jean-Marie Mégevanda ${ }^{a}$ Jean-Marc Heinicke ${ }^{b}$, François Pralong ${ }^{c}$ \\ a Dr., Chefarzt, Abteilung für Viszeralchirurgie, Hôpital de la Tour; ${ }^{b}$ Dr., Chefarzt, Abteilung für Viszeralchirurgie, Hôpital de la Tour;
}

c Prof., Chefarzt, Zentrum für Endokrinologie, Diabetologie und Adipositas, Hôpital de la Tour

In den letzten zwanzig Jahren hat sich der Anteil adipöser Menschen an der Bevölkerung beinahe verdoppelt. Dies hat nicht nur Einbussen an Lebenserwartung und -qualität bei den Betroffenen zur Folge, sondern verursacht auch Kosten in Höhe von 8 Milliarden Franken für die Gesellschaft. Direkte Behandlungen (einschliesslich Operationen) machen kaum ein Prozent dieser Ausgaben aus, reduzieren aber den Hauptkostenfaktor - die Komorbiditäten - und verbessern das Leben der Patienten.

\section{Adipositas: ein chronisches Leiden mit zahlreichen Komorbiditäten}

Frau S. stellte sich vor fünf Jahren erstmals in unserer Sprechstunde vor. Sie war damals 38 Jahre alt und von ihrem Hausarzt an uns überwiesen worden, um ihre Adipositas zu behandeln. Nach einer sechs Jahre zurückliegenden Scheidung hatte sie ihre Tätigkeit als Pflegeassistentin wiederaufnehmen müssen und zog alleine ihre beiden zwölf und zehn Jahre alten Kinder auf. Während der Schwangerschaften hatte sie jeweils etwa fünfzehn Kilogramm zugenommen und dieses Gewicht nicht wieder verloren. Nach ihrer Scheidung nahm sie weiter zu, bis zu den 120 Kilogramm, die sie wog, als sie zum ersten Mal in unsere Sprechstunde kam. Sie hatte mehrere Diäten und Jo-Jo-Effekte hinter sich, zweimal hatte sie zunächst jeweils rund zehn Kilogramm abgenommen und anschliessend rasch wieder zugelegt. Ihr Gewicht begann sie bei der Arbeit und im Alltag zu behindern. Sie litt unter einer behandelten arteriellen Hypertonie, einem SchlafapnoeSyndrom, das mit einer nächtlichen Atemunterstützung behandelt wurde, sowie einem unbehandelten Prädiabetes.

\section{Adipositas: mehr als eine Heraus- forderung Einzelner}

Obwohl die Patienten komplexe psychologische und medizinische Probleme aufweisen, wurde Adipositas erst 2006 von der Weltgesundheitsorganisation als ei- genständige Krankheit anerkannt. Aus diesem Grund lehren unsere Medizinfakultäten erst seit Kurzem spezifisch zu dieser Pathologie, deren Bedeutung sich aus den mit Adipositas verbundenen Komplikationen ergibt, wie etwa Diabetes, Atemprobleme während des Schlafs, erhöhte Cholesterinwerte, Bluthochdruck, Gelenkschmerzen und bestimmte Krebsarten. Es bedarf des Fachwissens und der Mitarbeit unterschiedlichster Gesundheitsberufe - aus den Bereichen Diätassistenz, Psychologie, Gastroenterologie, Pneumologie, Endokrinologie/Diabetologie, Chirurgie, Sportmedizin und Physiotherapie -, um diese Patienten dabei zu unterstützen, ihr Gewicht in den Griff zu bekommen sowie um allfällige Folgeerkrankungen zu erkennen und zu behandeln.

Ein solcher interdisziplinärer Ansatz erfordert zusätzliche Mittel, bildet aber einen der Schlüssel zu einer erfolgreichen Behandlung. Dabei wird der Patient in den Mittelpunkt des Gesundheitsnetzwerkes gestellt und wirkt bei der Behandlung seiner Krankheit und ihrer schädlichen Auswirkungen mit. Am Lebensweg unserer Patientin zeigt sich gut, wie wichtig dieser multimodale und umfassende Ansatz ist. Andernfalls setzt man diese Patienten wiederholten Jo-Jo-Episoden und einer kontinuierlichen Gewichtszunahme über die Jahre hinweg aus, was mit verringerter Lebensqualität und -erwartung einhergeht.

Das Hauptziel ist auf lange Sicht - und insbesondere bei jungen Patienten - die Gewichtsabnahme. Änderungen am Lebensstil können zwar das Risiko von Herz-Kreislauf-Erkrankungen erheblich senken und 
die Lebensqualität der Patienten deutlich erhöhen, in den meisten Fällen wirken sie sich aber nur mässig auf das Gewicht aus. Wenn sie angewendet werden kann, stellt die Adipositaschirurgie den einzigen Therapieansatz dar, mit dem krankhaft adipöse Patienten erheblich und dauerhaft Gewicht verlieren können.

\section{Die Adipositaschirurgie reduziert die Kosten für die Behandlung der Folgeerkrankungen und ihrer Auswirkungen deutlich.}

Die für die Schweiz verfügbaren Daten zur öffentlichen Gesundheit [1] bestätigen, dass dieser Ansatz auch wirtschaftlich sinnvoll ist. Die Ausgaben für Adipositasbehandlungen stellen etwa ein Prozent der Gesamtkosten dar, die durch Krankheiten im Zusammenhang mit Übergewicht und Adipositas verursacht werden. Die Adipositaschirurgie hingegen ermöglicht es, die Kosten für die Behandlung der Folgeerkrankungen von Adipositas oder ihrer Auswirkungen (Produktivitätseinbussen, Fehlzeiten, Berufsunfähigkeit) deutlich zu senken - und diese machen 99 Prozent der Gesamtkosten aus. Somit ist dieses Geld sehr gut investiert.

\section{Die Wirksamkeit eines Magenbypasses}

Das seit Anfang der 1990er Jahre immer weiterentwickelte chirurgische Verfahren des Magenbypasses ist heute sehr ausgereift: Risiko-Nutzen-Profil und das Verhältnis von Kosten und Wirksamkeit sprechen eindeutig für diesen Eingriff. Dies wird durch verschiedene Indikatoren belegt, die in gross angelegten prospektiven Studien ermittelt wurden. Besonders profitieren von einem solchen Eingriff junge Patienten sowie solche mit metabolischen Störungen wie in dem von uns beschriebenen Fall. Frau S. erhielt ein Jahr nach der ersten Konsultation und nach einer psychologischen und ernährungstechnischen Vorbereitung einen Magenbypass.

Vor dem Eingriff musste sie eine orale Antidiabetesbehandlung beginnen. Der Eingriff führte zu einem Gewichtsverlust von ca. 35 Kilogramm im ersten Jahr, begleitet von einer Normalisierung ihrer Blutdruckund Blutzuckerwerte, weshalb sie sämtliche Medikamente absetzen konnte. Die Nebenwirkungen des Eingriffs waren gering: Gelegentlich kam es unmittelbar nach dem Essen zu Unwohlsein, was sich durch eine Anpassung der Ernährung abstellen liess. Damit entspricht Frau S. der überwiegenden Mehrheit der Ope- rierten: Sie verlieren im Anschluss an eine Adipositaschirurgie einen Grossteil ihres Übergewichts und profitieren von einem anhaltenden Rückgang der damit verbundenen Komorbiditäten. Dies führt dazu, dass die Patienten auf die Einnahme einer Reihe von Medikamenten verzichten können und bei ihnen ein deutlicher Rückgang des kardiovaskulären Risikos und der globalen Mortalität verzeichnet werden kann.

Fünf Jahre nach dem Eingriff hält Frau S. mühelos ein Gewicht von rund 90 Kilogramm, da sie wieder regelmässig sportliche Aktivitäten ausüben kann. Sie arbeitet wieder ohne Probleme, ihr Schlafapnoe-Syndrom ist verschwunden, und sie konnte ihre Blutdrucksenker und ihre Medikamente zur Diabetesbehandlung absetzen. Sie benötigt Eisen-, Kalzium- und Vitamin-DPräparate, nimmt täglich eine Multivitamintablette und weist eine durch den Magenbypass erheblich verbesserte Lebensqualität auf.

\section{Fazit}

Die Geschichte dieser Patientin ist insofern bemerkenswert, als sie die Vorteile des chirurgischen Eingriffs und der interdisziplinären Betreuung sehr gut veranschaulicht. Diese Vorteile zeigen sich nicht nur im besseren Gesundheitszustand und der höheren Lebensqualität der Patientin, sondern auch im Hinblick auf die Gesellschaft: Die Patientin arbeitet nun wieder Vollzeit, und nach dem Verschwinden der Begleiterscheinungen entsprechen ihr Herz-Kreislauf-Risiko und ihre Lebenserwartung wieder dem der nicht adipösen Bevölkerung.

\section{Zusammenfassung}

Wir beschreiben eine Patientin von 38 Jahren, die unter morbider Adipositas mit mehreren Folgeerkrankungen litt, was ihr erschwerte, ihrer Arbeit als Pflegeassistentin nachzugehen. Nach entsprechender Vorbereitung erhielt sie einen Magenbypass, in dessen Folge sie 35 Kilogramm verlor. Heute nimmt sie keinerlei Medikamente, arbeitet wieder Vollzeit und geniesst dieselbe Lebensqualität wie vor ihrer Gewichtszunahme: Nicht nur der Patientin geht es heute besser, auch die direkten wie indirekten Kosten ihrer morbiden Adipositas konnten gesenkt werden.

Literatur

1 Bundesamt für Gesundheit; Indikator 5.2: Kosten von Übergewicht und Adipositas (PDF, $101 \mathrm{kB}, 19.6 .2017$ ). 